\title{
Artigo \\ Custos catastróficos e sequelas sociais decorrentes do diagnóstico e tratamento da tuberculose no Brasil $^{*}$
}

\section{Catastrophic costs and social sequels due to tuberculosis diagnosis and treatment in Brazil}

\author{
Costos catastróficos y consecuencias sociales resultantes del diagnóstico y tratamiento de la \\ tuberculosis en Brasil
}

\author{
Leticia Molino Guidoni' - — orcid.org/0000-0003-3737-5407 \\ Eliana Zandonade ${ }^{2}$ - @orcid.org/0000-0001-5160-3280 \\ Geisa Fregona ${ }^{2}$ - @ orcid.org/0000-0001-5835-8222 \\ Letícya dos Santos Almeida Negri' - @ orcid.org/0000-0002-0056-4506 \\ Sandra Maria do Valle Leone de Oliveira ${ }^{3}$ - Đ orcid.org/0000-0002-8960-6716 \\ Thiago Nascimento do Prado' - @ orcid.org/0000-0001-8132-6288 \\ Carolina Maia Martins Sales' 1 - @ orcid.org/0000-0002-2879-5621 \\ Raisa da Silveira Coimbra' - (D orcid.org/0000-0003-2528-6044 \\ Heletícia Scabelo Galavote - Đ orcid.org/0000-0003-4490-6763 \\ Ethel Leonor Noia Maciel ${ }^{2}$ - @ orcid.org/0000-0003-4826-3355 \\ 'Universidade Federal do Espírito Santo, Laboratório de Epidemiologia, Vitória, ES, Brasil \\ Universidade Federal do Espírito Santo, Programa de Pós-Graduação em Saúde Coletiva, Vitória, ES, Brasil \\ ${ }^{3}$ Universidade Federal de Mato Grosso do Sul, Programa de Pós-Graduação em Doenças Infecciosas e Parasitárias, Campo Grande, MS, Brasil \\ ${ }^{4}$ Universidade Federal do Espírito Santo, Departamento de Ciências da Saúde, São Mateus, ES, Brasil
}

\section{Resumo}

Objetivo: Avaliar o impacto dos custos catastróficos no desfecho desfavorável do tratamento da tuberculose. Métodos: Estudo de coorte prospectiva, realizado em cinco capitais de estados brasileiros (Manaus, Recife, Vitória, Campo Grande e Porto Alegre) no período de junho de 2016 a julho de 2018. Empregou-se regressão logística para calcular a razão de chances (odds ratio, OR) e os intervalos de confiança de 95\% ( IC $_{95 \%}$ ). Resultados: Dos 350 participantes, 310 foram incluídos, dos quais 30 apresentaram desfecho desfavorável. Custo catastrófico $\left(\mathrm{OR}=2,53-\mathrm{IC}_{95 \%} 1,13 ; 5,67\right)$ e divórcio $\left(\mathrm{OR}=5,29-\mathrm{IC}_{95 \%} 1,39 ; 20,05\right)$ aumentaram as chances de desfecho desfavorável. Conclusão: Dificuldades financeiras durante o tratamento da tuberculose podem prejudicar seu desfecho. Os fatores determinantes para o desfecho do tratamento foram custo catastrófico e divórcio.

Palavras-chave: Tuberculose; Terapêutica; Custos e Análise de Custo; Estudos de Coortes; Fatores Socioeconômicos; Brasil.

*Artigo derivado da tese de doutorado intitulada 'Impacto dos custos catastróficos no tratamento de pacientes com tuberculose em cinco regiões do Brasil', defendida por Leticia Molino Guidoni junto ao Programa de Pós-Graduação em Saúde Coletiva da Universidade Federal do Espírito Santo em 10 de agosto de 2020.

Endereço para correspondência:

Leticia Molino Guidoni - Rodovia Governador Mário Covas, Km 60 - Bairro Litorâneo, São Mateus, ES, Brasil. CEP 29932-540

E-mail: leticiaguidoni@hotmail.com 


\section{Introdução}

A tuberculose é uma doença infecciosa com forte determinante social, intimamente ligada à pobreza, com importante impacto socioeconômico. ${ }^{1-6} \mathrm{Em}$ 2015, a Organização Mundial da Saúde (OMS) estimou cerca de 10,4 milhões de novos casos e 1,4 milhão de mortes por tuberculose no mundo. Em 2016, eram 11,4 milhões de novos casos e 1,6 milhão de mortes apenas nos países em desenvolvimento. ${ }^{7}$ Os gastos com prevenção, diagnóstico e tratamento da doença alcançaram US\$ 6,6 bilhões em 2015, superando os US\$ 6,2 bilhões gastos em $2014 .^{8}$ Atualmente, as estimativas para o financiamento do controle da tuberculose nos 129 países de baixa e média renda monitorados pelo plano global para acabar com a tuberculose vêm aumentando e, para o quinquênio 2018-2022, estão previstos US\$ 60 bilhões, à média de US\$ 12 bilhões anuais. ${ }^{9}$

\section{Nenhuma pessoa com tuberculose ou seus familiares deve enfrentar custos catastróficos decorrentes da doença, definidos como dispêndios excedentes a $20 \%$ da renda familiar anual, de acordo com a Organização Mundial da Saúde.}

o Brasil ocupa o $30^{\circ}$ lugar na lista dos países com maior carga de tuberculose. ${ }^{9}$ No país, a cada ano são notificados, aproximadamente, 80 mil casos; em 2019, foram 73.864 casos incidentes. ${ }^{10}$ Entre os fatores que mais contribuem para esse cenário estão a pobreza e a grande densidade populacional dos centros urbanos. ${ }^{11}$

A OMS propôs metas para a eliminação da tuberculose no mundo até $2035 .{ }^{12}$ A nova estratégia para o fim da tuberculose definiu três indicadores de alto nível, para facilitar o progresso no sentido de se eliminar a infecção. Uma dessas metas é a de que nenhuma pessoa com tuberculose ou seus familiares deve enfrentar custos catastróficos decorrentes da doença. ${ }^{13,14}$

No Brasil, apesar de o Sistema Único de Saúde (SUS) oferecer tratamento universal e gratuito para a tuberculose,$^{15}$ a busca por atendimento, diagnóstico e acompanhamento durante 0 tratamento representa gastos que podem levar a prejuízos e agravamento da situação econômico-financeira, e até mesmo empobrecimento de muitas famílias. ${ }^{16}$
Os custos totais enfrentados pelas pessoas com tuberculose incluem despesas diretas com medicamentos, intervenções hospitalares e médicas, exames e transporte. Os custos indiretos incluem, principalmente, a perda de renda causada pela incapacidade para o trabalho durante o adoecimento. Custos diretos e indiretos podem ocorrer antes e após o diagnóstico de tuberculose, e afetar tanto os indivíduos doentes como outros membros da família que lhes prestam cuidados e apoio..$^{13}$ Além dos custos diretos e/ou indiretos, os residentes nos domicílios atingidos pela tuberculose podem ver-se obrigados ao uso de recursos de poupanças financeiras e tomadas de empréstimo. ${ }^{13}$

Estudos que caracterizam os custos relacionados com a tuberculose no domicílio já foram realizados em outros países..$^{1-3,16-18}$ No Brasil, são poucas as pesquisas dessa natureza, normalmente focadas em uma cidade ou um estado. ${ }^{19-21}$

0 presente estudo teve por objetivo avaliar 0 impacto econômico em nível familiar e sequelas sociais observadas, a partir do diagnóstico e tratamento da tuberculose no Brasil.

\section{Métodos}

Trata-se de um estudo de coorte prospectiva, sobre dados coletados mediante entrevista presencial e avaliação do encerramento de casos notificados no Sistema de Informação de Agravos de Notificação (Sinan).

A pesquisa foi realizada em cinco capitais de estados brasileiros - Manaus/AM, Recife/PE, Vitória/ES, Campo Grande/MS, e Porto Alegre/RS -, cujos dados foram colhidos em 14 unidades de saúde distribuídas entre as cinco grandes regiões do país, no período de junho de 2016 a julho de 2018. As unidades de saúde selecionadas oferecem tratamento para tuberculose conforme as orientações da Coordenação-Geral de Vigilância das Doenças de Transmissão Respiratória de Condições Crônicas (CGDR), do Ministério da Saúde.

A amostra analisada foi composta por indivíduos diagnosticados com tuberculose, de idade superior a 18 anos, em tratamento há pelo menos um mês com esquema básico (rifampicina, isoniazida, pirazinamida e etambutol por dois meses, e rifampicina e isoniazida por mais quatro meses). 0 primeiro acompanhamento foi realizado localmente, por meio de entrevista com indivíduos em tratamento para tuberculose nas unidades selecionadas, por demanda livre, ou seja: quem 
aceitou ser entrevistado foi incluído como participante da pesquisa. No segundo acompanhamento, realizou-se a avaliação dos dados de desfecho do tratamento descrito em epígrafe, disponibilizados pelas coordenações municipais de vigilância à medida que os casos de tuberculose eram encerrados.

Foram analisadas as seguintes variáveis:

a) Sociodemográficas

- Sexo (masculino, feminino);

- Escolaridade (em anos de estudo: 0-8; >8);

- Idade (em anos: 18-39; $\geq 40$; não informado);

- Raça/cor da pele (branca; não branca [preta; amarela; parda; indígena; não informada]);

- Chefe da família com tuberculose (sim; não);

- Emprego formal (sim; não);

- Interrupção do trabalho ou estudo (sim; não; não informado);

- Plano de saúde (sim; não).

b) Clínicas

- Tipo de tuberculose (pulmonar; extrapulmonar);

- Fase do tratamento (intensiva; de continuação);

- HIV (positivo; negativo; não testado/desconhecido);

- Hospitalização (sim; não);

- Tratamento anterior de tuberculose (sim; não);

- Primeiro atendimento (público; privado; outros);

- Comorbidades (sim; não).

c) Alterações familiares durante 0 adoecimento por tuberculose

- Sequela social (sim; não);

- Abandono escolar (sim; não);

- Abandono do trabalho (sim; não);

- Divórcio (sim; não);

- Perturbação da vida sexual (sim; não);

- Adoecimento de filho (sim; não);

- Afastamento das pessoas (sim; não);

- Depressão (sim; não);

- Experiência de preconceito devido à tuberculose

(sim; não);

- Outras sequelas sociais (sim; não).

d) Condições econômicas

- Condição financeira do domicílio antes da tuberculose (pobre; não pobre);

- Condição financeira do domicílio depois da tuberculose (pobre; não pobre);

- Alteração de renda do domicílio após a tuberculose (sim; não).

e) Exposição
- Custo catastrófico superior a 20\% da renda familiar anual antes da tuberculose (sim; não).

f) Desfecho do tratamento (desfecho favorável [cura; tratamento completo]; desfecho desfavorável [óbito; abandono; falência de tratamento]).

Considerou-se 'custos catastróficos' o equivalente aos dispêndios das famílias quando excedem $20 \%$ da renda familiar anual, de acordo com o preconizado pela OMS. ${ }^{13,14}$ Adotou-se, como instrumento para a coleta de dados, o 'Tool to Estimate Patient's Costs' da OMS, em sua versão traduzida e adaptada à cultura do Brasil. ${ }^{22,23} \mathrm{~A}$ mensuração de custo catastrófico partiu da soma de custos totais, dividida pelo valor da renda familiar anual antes do adoecimento por tuberculose (renda familiar mensal multiplicada por 12).

Para estimar os custos diretos médicos e não médicos relacionados com a tuberculose, utilizou-se 0 total de custos diretos médicos durante a fase de pré-diagnóstico e diagnóstico (a soma dos custos totais administrativos, custos com testes diagnósticos, com exame radiológico e com medicamentos); e para os custos diretos não médicos, os gastos com viagem, alimentação e acomodação - desconsiderados quaisquer seguros de reembolso.

A abordagem para valoração utilizada foi a de microcusteio. ${ }^{24} 0$ cálculo do custo total direto durante 0 tratamento considerou:

a) custo para visitas aos serviços de saúde, necessárias ao tratamento, diretamente observado por mês (custos com transporte e alimentação para cada visita semanal, assumindo-se quatro semanas em um mês);

b) custo com a retirada de medicamentos (soma dos custos com transporte e alimentação, custos administrativos e com acomodação, multiplicada pelo número de visitas ao serviço/mês);

c) custo de acompanhamento (soma do custo total de uma visita com honorários, exames de escarro, exame radiológico e medicamentos, entre outros, multiplicada pelo número de visitas a serviço de saúde/mês);

d) custo com hospitalização (soma dos honorários com administração hospitalar, lençóis/rouparia, alimentação, transporte, medicamentos, exames e outros); e

e) custo mensal com aquisição de alimentos especiais.

A abordagem para valoração de custos indiretos adotou o método de capital humano. Neste caso, para 
a mensuração de custos indiretos relacionados com a tuberculose, utilizou-se o total de custos indiretos do indivíduo infectado, motivo de incapacidade para 0 trabalho. ${ }^{24} 0$ cálculo da incapacidade de trabalho partiu do rendimento individual mensal antes da tuberculose, multiplicado pelo número de meses sem poder trabalhar por conta do adoecimento. A perspectiva abordada foi a do paciente como pagador. ${ }^{24}$

Utilizou-se a mensuração de extrapolamento para estimar os custos das fases em que os participantes não tiveram os dados coletados (fase intensiva e de manutenção). Para entrevistados na fase intensiva, utilizou-se a soma do custo do pré-diagnóstico e diagnóstico mais custos diretos (médicos e não médicos) e custos indiretos, multiplicada por 2 e somada à média dos custos da fase de manutenção, finalmente multiplicada por 4 ou 6 (a depender do número de meses previstos para o tratamento). Relativamente aos participantes entrevistados na fase de manutenção, utilizou-se a soma do custo do pré-diagnóstico e diagnóstico mais custos diretos (médicos e não médicos) e custos indiretos, multiplicada por 4 ou 6 (a depender do número de meses previstos para 0 tratamento) e somada à média dos custos da fase intensiva, finalmente multiplicada por 2. Portanto, para o custo total com extrapolamento, utilizou-se o valor de custo mensal de acordo com a fase em que o participante foi entrevistado, multiplicado pelo número de meses em que essa fase foi estabelecida para o tratamento do participante mais 0 valor de extrapolamento determinado para a fase em que o participante não foi entrevistado.

Para o cálculo do empobrecimento (redução da renda do participante durante 0 tratamento para tuberculose), utilizou-se a renda mensal antes do adoecimento dividida pelo número de moradores da residência. Para caracterização de pobreza, adotou-se o limite superior estabelecido pelo Banco Mundial, de $\mathbf{R} \$ 387,07$ por mês como definição de uma pessoa pobre. ${ }^{25}$ Assim, uma renda inferior a $\mathrm{R} \$ 387,07 / \mathrm{mês}$ por morador foi considerada condição 'pobre', e uma renda superior a essa, 'não pobre'.

$\mathrm{Na}$ abordagem dos participantes da pesquisa, visando minimizar eventual viés recordatório dos gastos autorreferidos e, assim, instruir as respostas ao questionário, o pesquisador encarregado da coleta dos dados adotou medidas didáticas específicas, a saber: auxílio de calendários; menção das últimas visitas às unidades de saúde; e valores gastos na última compra, realizada no mês ou semana em curso.

0 tamanho do estudo foi calculado pelo programa Epidat ${ }^{\circledR}$ 4.0. Considerou-se como fator de exposição o custo catastrófico, e como desfecho, um desfecho desfavorável (óbito, abandono ou falência do tratamento). Arbitrariamente, considerou-se (i) o risco entre os expostos de 15\%, e entre os não expostos, de $7 \%$, (ii) a razão entre não expostos e expostos de 5 , (iii) nível de significância de 5\% e (iv) um poder do teste de $85 \%$. Os tamanhos mínimos foram definidos em 151 indivíduos sem custos catastróficos e 75 com custos catastróficos. Prevendo-se possíveis perdas, fosse durante a obtenção dos dados de custos, fosse no desfecho, aumentou-se a amostra em 55\%, obtendo-se um tamanho amostral de 350 participantes, divididos de acordo com a incidência de cada capital selecionada: Recife (29,3\%; 103 participantes); Campo Grande $(5,1 \% ; 18$ participantes); Vitória $(2,1 \% ; 7$ participantes); Manaus (36\%; 126 participantes); e Porto Alegre (27,3\%; 96 participantes).

$\mathrm{Na}$ descrição das variáveis, foram calculadas as frequências absolutas e relativas. $\mathrm{Na}$ análise bivariável, adotou-se o teste qui-quadrado de Pearson e 0 texto exato de Fisher.

Os resultados das análises de regressão logística foram expressos como razão de chances (odds ratio, OR) e intervalos de confiança de $95 \%$ ( $\mathrm{IC}_{95 \%}$ ). A regressão logística serviu para investigar fatores de risco do desfecho do tratamento da tuberculose. Fatores de risco hipotéticos associados aos desfechos em valor $\mathrm{p}<0,2$ foram incluídos na análise multivariável. Para a análise múltipla, empregou-se um modelo teórico de classificação dos determinantes em níveis hierárquicos: no nível distal, variáveis sociodemográficas; no nível intermediário, informações clínicas do atual tratamento da tuberculose e alterações familiares durante o adoecimento por tuberculose; e no nível proximal, variáveis financeiras. Valores de $\mathrm{p}<0,05$ foram considerados estatisticamente significativos. Para análise estatística dos dados, aplicou-se o software de estatística Stata 14.0.

O projeto do estudo foi aprovado pelo Comitê de Ética em Pesquisa com Seres Humanos do Centro de Ciências da Saúde da Universidade Federal do Espírito Santo (CEP/CCS/UFES): Parecer $n^{\circ}$ 1.856.319, emitido em 7 de dezembro de 2016; Certificado de Apresentação para 
Apreciação Ética (CAAE) nº 61080416.7.0000.5060. Todos os participantes foram informados sobre os objetivos da pesquisa e assinaram o Termo de Consentimento Livre e Esclarecido, antes de conceder entrevista.

\section{Resultados}

Foram elegíveis, no primeiro acompanhamento do estudo, 361 participantes, dos quais 11 não informaram renda familiar antes do adoecimento pela tuberculose, não sendo possível o cálculo do custo catastrófico para eles, enquanto outros 40 (11\%) não tinham o registro do desfecho do tratamento no Sinan. A amostra final foi de 310 participantes (Figura 1).

Das pessoas em tratamento para tuberculose participantes, $65 \%$ eram do sexo masculino e $46 \%$ chefes da família. Em relação a trabalho, $60 \%$ dos entrevistados se encontravam desempregados ou estavam inseridos no mercado de trabalho de maneira informal; 62\% deixaram de trabalhar, estudar ou fazer atividades domésticas devido ao adoecimento. Dos entrevistados, $83 \%$ eram casos novos de tuberculose, e dos participantes em retratamento, $44 \%$ não completaram o tratamento anterior de tuberculose. Dos domicílios estudados, $71 \%$ foram considerados não pobres antes do adoecimento por tuberculose de um de seus membros; esse percentual reduziu-se para $61 \%$ após o adoecimento, demonstrando uma redução de renda em 11\% dos domicílios estudados (Tabela 1).

De acordo com a Tabela 2, 30 participantes apresentaram desfecho desfavorável. $\mathrm{Na}$ análise ajustada, o divórcio $\left(\mathrm{OR}=5,29-\mathrm{IC}_{95 \%}, 1,39 ; 20,05\right)$ e 0 custo catastrófico $\left(\mathrm{OR}=2,53-\mathrm{IC}_{95 \%} 1,13 ; 5,67\right)$ aumentaram significativamente as chances de desfecho desfavorável ao tratamento.

\section{Discussão}

As dificuldades financeiras enfrentadas pelas famílias no Brasil, durante o diagnóstico e tratamento por tuberculose, e as sequelas sociais da doença, influenciam no desfecho do tratamento. Dos participantes incluídos e acompanhados durante 0 tratamento da tuberculose, a maioria que apresentou desfecho desfavorável também referiu custo catastrófico. Esta é a primeira pesquisa realizada no Brasil com indivíduos tratados para tuberculose e dedicada à análise de associação entre custos catastróficos e desfecho do tratamento experimentado pelo doente e outras pessoas de seu domicílio.

A análise baseou-se nos gastos autorreferidos, sujeitos a subestimação ou superestimação pelos participantes. Coletaram-se dados de homens e mulheres com tuberculose atendidos pelos serviços de diagnóstico e tratamento da doença, portanto sem captar aqueles que não buscaram tratamento. Realizou-se a coleta dos dados a partir do segundo mês de tratamento e, portanto, o viés de memória pode ter afetado as descobertas. 0 caráter gratuito do tratamento da tuberculose no Brasil, entretanto, impediu captar alguns gastos hospitalares, limitando a comparação dos dados da análise com outros cenários. Participantes que atuam na economia informal, autônomos e em atividade agrícola encontram dificuldades em aferir rendimentos e, por conseguinte, os resultados de desfecho do tratamento de alguns deles não foram obtidos, dada a limitação do estudo ao uso dos dados secundários. Para este artigo, os dados de estratificação de custos não foram apresentados, embora, acredita-se, essas limitações não tenham interferido nos resultados.

Aproximadamente dois quintos dos participantes referiram custos catastróficos, achado semelhante ao de estudos realizados na Indonésia e em Uganda, onde o custo catastrófico foi $36 \%{ }^{4}$ e $53 \%$ respectivamente. ${ }^{3} 0$ principal contribuinte para os custos totais é a perda de renda do participante ao adoecer por tuberculose. Um atributo relevante a considerar é o longo tempo de tratamento e a gravidade de alguns casos, o que pode levar a incapacidade maior ou menor de retomada das atividades laborais. Assim como em outros estudos, o impacto social da tuberculose em indivíduos e suas famílias foi alarmante, consequência da perda significativa de renda e do custo gerado pelo diagnóstico e tratamento. ${ }^{26,27}$ Participantes de outras pesquisas também afirmam que foram afetados com a perda de renda e de trabalho. ${ }^{16,26}$

Dos participantes com desfecho desfavorável, a maioria apresentou custo catastrófico. A chance de indivíduos que tiveram tuberculose apresentarem custo catastrófico e não obterem êxito no tratamento foi, aproximadamente, 2,5 vezes maior comparada à dos que não apresentaram custo catastrófico. A despeito de o Brasil contar com uma cobertura universal dos serviços de saúde do SUS específica para o diagnóstico e tratamento da tuberculose, a doença implica outros custos, resultantes da incapacidade de trabalho no 


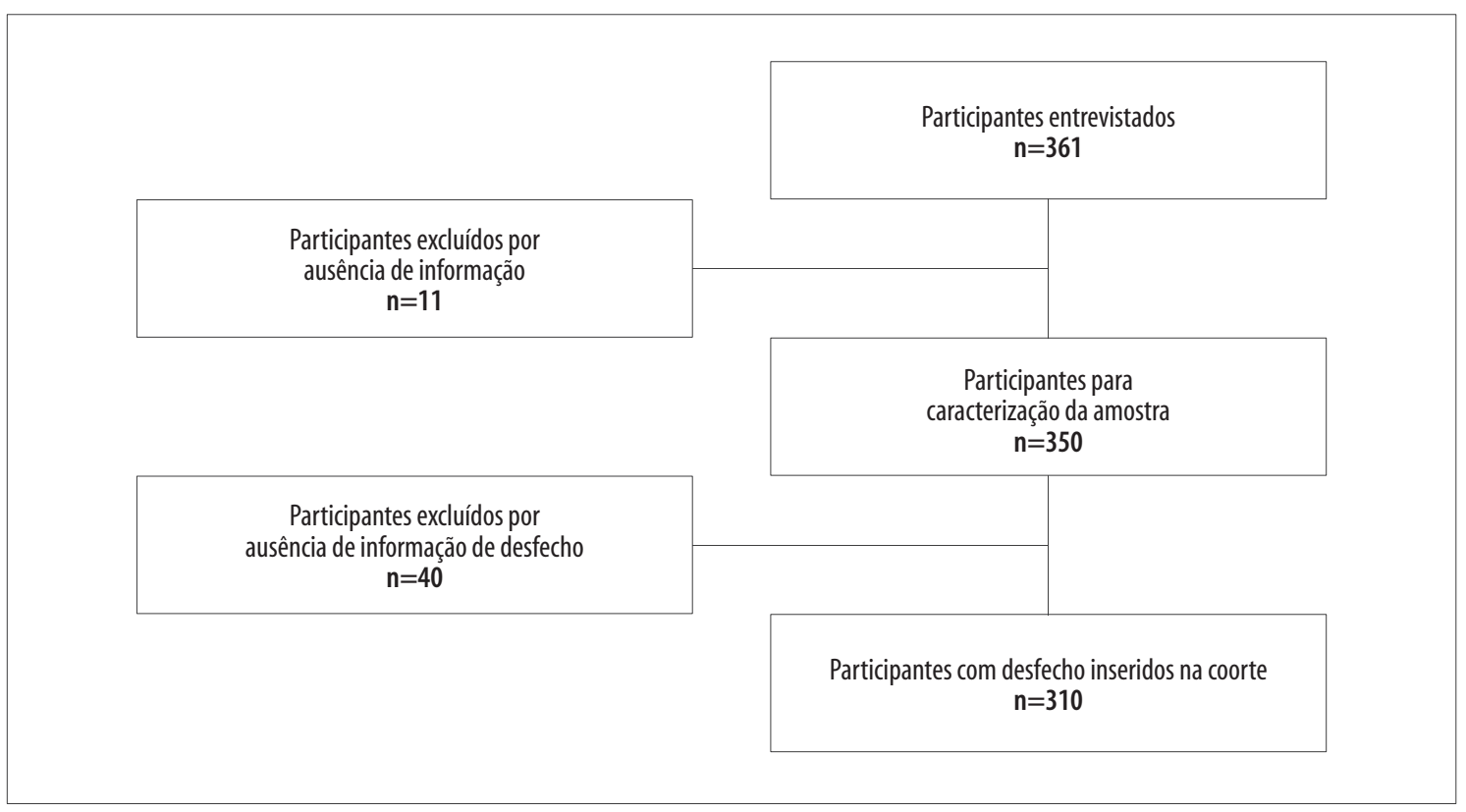

Figura 1 - Processo de inclusão dos participantes na pesquisa

Tabela 1 - Distribuição das características sociodemográficas e clínicas, alterações familiares durante 0 adoecimento e condição econômica, de acordo com o custo catastrófico por tuberculose dos participantes com tuberculose das cinco capitais ( $n=350)$, Brasil, junho/2016-julho/2018

\begin{tabular}{|c|c|c|c|}
\hline Variáveis & $\begin{array}{l}\mathrm{N}(\%) \\
\text { Total }\end{array}$ & $\begin{array}{c}\mathrm{N}(\%) \\
\text { Sem custo } \\
\text { catastrófico }\end{array}$ & $\begin{array}{c}\mathrm{N}(\%) \\
\text { Com custo } \\
\text { catastrófico }\end{array}$ \\
\hline \multicolumn{4}{|l|}{ Sexo } \\
\hline Masculino & $226(65)$ & $132(58)$ & $94(42)$ \\
\hline Feminino & $124(35)$ & $73(59)$ & $51(41)$ \\
\hline \multicolumn{4}{|c|}{ Escolaridade (anos de estudo) } \\
\hline $0-8$ & $173(49)$ & $82(47)$ & $91(53)$ \\
\hline$>8$ & $177(51)$ & $123(69)$ & $54(31)$ \\
\hline \multicolumn{4}{|l|}{ Idade (anos) } \\
\hline $18-39$ & $165(47)$ & $90(55)$ & $75(45)$ \\
\hline$\geq 40$ & $182(52)$ & $113(62)$ & $69(38)$ \\
\hline Não informado & $3(1)$ & $2(67)$ & $1(33)$ \\
\hline \multicolumn{4}{|c|}{ Raça/cor da pele } \\
\hline Branca & $91(26)$ & $59(65)$ & $32(35)$ \\
\hline Não branca & $257(73)$ & $145(56)$ & $112(44)$ \\
\hline Não informada & $2(1)$ & $1(50)$ & $1(50)$ \\
\hline \multicolumn{4}{|c|}{ Chefe da família com tuberculose } \\
\hline Sim & $162(46)$ & $86(53)$ & $76(47)$ \\
\hline Não & $188(54)$ & $119(63)$ & $69(37)$ \\
\hline
\end{tabular}

a) HIV:vírus da imunodeficiência humana (sigla em inglês para human immunodeficiency virus). 
Continuação

Tabela 1-Distribuição das características sociodemográficas e clínicas, alterações familiares durante 0 adoecimento e condição econômica, de acordo com o custo catastrófico por tuberculose dos participantes com tuberculose das cinco capitais ( $n=350)$, Brasil, junho/2016-julho/2018

\begin{tabular}{|c|c|c|c|}
\hline Variáveis & $\begin{array}{l}\mathrm{N}(\%) \\
\text { Total }\end{array}$ & $\begin{array}{c}\mathrm{N}(\%) \\
\text { Sem custo } \\
\text { catastrófico }\end{array}$ & $\begin{array}{c}\mathrm{N}(\%) \\
\text { Com custo } \\
\text { catastrófico }\end{array}$ \\
\hline \multicolumn{4}{|l|}{ Emprego formal } \\
\hline $\operatorname{Sim}$ & $139(40)$ & $105(76)$ & $34(24)$ \\
\hline Não & $211(60)$ & $100(47)$ & $111(53)$ \\
\hline \multicolumn{4}{|c|}{ Interrupção do trabalho ou estudo } \\
\hline Sim & $218(62)$ & $104(48)$ & $114(52)$ \\
\hline Não & $126(36)$ & $96(76)$ & $30(24)$ \\
\hline Não informado & $6(1)$ & $5(83)$ & $1(18)$ \\
\hline \multicolumn{4}{|l|}{ Plano de saúde } \\
\hline Sim & $73(21)$ & $62(85)$ & $11(15)$ \\
\hline Não & $277(79)$ & $143(52)$ & $134(48)$ \\
\hline \multicolumn{4}{|l|}{ Tipo de tuberculose } \\
\hline Pulmonar & $263(75)$ & $161(61)$ & $102(39)$ \\
\hline Extrapulmonar & $87(25)$ & $44(51)$ & $43(49)$ \\
\hline \multicolumn{4}{|l|}{ Fase do tratamento } \\
\hline Intensiva & $98(28)$ & $56(57)$ & $42(43)$ \\
\hline Continuação & $252(72)$ & $149(59)$ & $103(41)$ \\
\hline \multicolumn{4}{|l|}{ HIV $^{\mathrm{a}}$} \\
\hline Positivo & $60(17)$ & $26(43)$ & $34(57)$ \\
\hline Negativo & $241(69)$ & $149(62)$ & $92(38)$ \\
\hline Não testado/desconhecido & $49(14)$ & $30(61)$ & $19(39)$ \\
\hline \multicolumn{4}{|l|}{ Hospitalização } \\
\hline Sim & $140(40)$ & $65(46)$ & $75(54)$ \\
\hline Não & $210(60)$ & $140(67)$ & $70(33)$ \\
\hline \multicolumn{4}{|c|}{ Tratamento anterior de tuberculose } \\
\hline Sim & $60(17)$ & $30(50)$ & $30(50)$ \\
\hline Não & $290(83)$ & $175(60)$ & $115(40)$ \\
\hline \multicolumn{4}{|l|}{ Primeiro atendimento } \\
\hline Público & $314(90)$ & $180(57)$ & $134(43)$ \\
\hline Privado & $22(6)$ & $18(82)$ & $4(18)$ \\
\hline Outros & $14(4)$ & $7(50)$ & $7(50)$ \\
\hline \multicolumn{4}{|l|}{ Comorbidades } \\
\hline Sim & $142(41)$ & $76(54)$ & $66(46)$ \\
\hline Não & $208(59)$ & $129(62)$ & $79(38)$ \\
\hline \multicolumn{4}{|l|}{ Sequela social } \\
\hline Sim & $230(66)$ & $123(53)$ & $107(47)$ \\
\hline Não & $120(34)$ & $82(68)$ & $38(32)$ \\
\hline \multicolumn{4}{|l|}{ Abandono escolar } \\
\hline Sim & $5(1)$ & $3(60)$ & $2(40)$ \\
\hline Não & $345(99)$ & $202(59)$ & $143(41)$ \\
\hline
\end{tabular}

a) HIV: vírus da imunodeficiência humana (sigla em inglês para human immunodeficiency virus). 
Continuação

Tabela 1 - Distribuição das características sociodemográficas e clínicas, alterações familiares durante 0 adoecimento e condição econômica, de acordo com o custo catastrófico por tuberculose dos participantes com tuberculose das cinco capitais $(n=350)$, Brasil, junho/2016-julho/2018

\begin{tabular}{|c|c|c|c|}
\hline Variáveis & $\begin{array}{l}\mathrm{N}(\%) \\
\text { Total }\end{array}$ & $\begin{array}{c}N(\%) \\
\text { Sem custo } \\
\text { catastrófico }\end{array}$ & $\begin{array}{c}\mathrm{N}(\%) \\
\text { Com custo } \\
\text { catastrófico }\end{array}$ \\
\hline \multicolumn{4}{|c|}{ Abandono do trabalho } \\
\hline $\operatorname{Sim}$ & $98(28)$ & $40(41)$ & $58(59)$ \\
\hline Não & $252(72)$ & $165(65)$ & $87(35)$ \\
\hline \multicolumn{4}{|l|}{ Divórcio } \\
\hline Sim & $17(5)$ & $8(47)$ & $9(53)$ \\
\hline Não & $333(95)$ & $197(59)$ & $136(41)$ \\
\hline \multicolumn{4}{|c|}{ Perturbação da vida sexual } \\
\hline $\operatorname{Sim}$ & $10(3)$ & $7(70)$ & $3(30)$ \\
\hline Não & $340(97)$ & $198(58)$ & $142(42)$ \\
\hline \multicolumn{4}{|c|}{ Adoecimento de filho } \\
\hline Sim & $4(1)$ & $3(75)$ & $1(25)$ \\
\hline Não & $346(99)$ & $202(58)$ & $144(42)$ \\
\hline \multicolumn{4}{|c|}{ Afastamento das pessoas } \\
\hline $\operatorname{Sim}$ & $95(25)$ & - & $95(100)$ \\
\hline Não & $255(73)$ & $120(47)$ & $135(53)$ \\
\hline \multicolumn{4}{|l|}{ Depressão } \\
\hline $\operatorname{Sim}$ & $10(3)$ & $7(70)$ & $3(30)$ \\
\hline Não & $340(97)$ & $198(58)$ & $142(41)$ \\
\hline \multicolumn{4}{|c|}{ Experiência de preconceito devido à tuberculose } \\
\hline Sim & $17(5)$ & $12(71)$ & $5(29)$ \\
\hline Não & $333(95)$ & $193(58)$ & $140(42)$ \\
\hline \multicolumn{4}{|c|}{ Outras sequelas sociais } \\
\hline $\operatorname{Sim}$ & $128(37)$ & $78(61)$ & $50(39)$ \\
\hline Não & $222(63)$ & $127(57)$ & $95(43)$ \\
\hline \multicolumn{4}{|c|}{ Condição financeira do domicílio antes da tuberculose } \\
\hline Pobre & $101(29)$ & $33(33)$ & $68(67)$ \\
\hline Não pobre & $249(71)$ & $172(69)$ & $77(31)$ \\
\hline \multicolumn{4}{|c|}{ Condição financeira do domicílio depois da tuberculose } \\
\hline Pobre & $138(39)$ & $54(39)$ & $84(61)$ \\
\hline Não pobre & $212(61)$ & $151(71)$ & $61(29)$ \\
\hline \multicolumn{4}{|c|}{ Alteração de renda do domicílio após a tuberculose } \\
\hline Sim & $40(11)$ & $22(55)$ & $18(45)$ \\
\hline Não & $310(89)$ & $183(59)$ & 127 (41) \\
\hline Total & $350(100)$ & $205(59)$ & $145(41)$ \\
\hline
\end{tabular}

a) HIV:vírus da imunodeficiência humana (sigla em inglês para human immunodeficiency virus). 
Tabela 2 -Desfecho do tratamento para tuberculose e razão de chances do desfecho desfavorável pelas características dos participantes com tuberculose das cinco capitais ( $n=310)$, Brasil, junho/2016julho/2018

\begin{tabular}{|c|c|c|c|c|c|c|}
\hline \multirow[b]{2}{*}{ Variáveis } & \multicolumn{2}{|c|}{ Desfecho do tratamento } & \multirow{2}{*}{$\begin{array}{c}\text { Razão de chances } \\
\text { bruta } \\
\left(\mathrm{IC}_{95 \%}^{\mathrm{a}}\right)\end{array}$} & \multirow[b]{2}{*}{ p-valor } & \multirow{2}{*}{$\begin{array}{c}\text { Razão de chances } \\
\text { ajustada } \\
\left(\mathrm{IC}_{95 \%}{ }^{\mathrm{a}}\right)\end{array}$} & \multirow[b]{2}{*}{ p-valor } \\
\hline & $\begin{array}{l}\text { Favorável } \\
(\%)\end{array}$ & $\begin{array}{l}\text { Desfavorável } \\
(\%)\end{array}$ & & & & \\
\hline \multicolumn{7}{|c|}{ Nível distal: variáveis sociodemográficas } \\
\hline \multicolumn{7}{|l|}{ Sexo } \\
\hline Masculino & $182(91)$ & $18(9)$ & $0,80(0,35 ; 1,92)$ & $0,586^{d}$ & - & \\
\hline Feminino & $98(89)$ & $12(11)$ & & & & \\
\hline \multicolumn{7}{|c|}{ Escolaridade (anos de estudo) } \\
\hline $0-8$ & $145(91)$ & $14(9)$ & $1,22(0,53 ; 2,82)$ & $0,594^{d}$ & - & \\
\hline$>8$ & $135(89)$ & $16(11)$ & & & & \\
\hline \multicolumn{7}{|l|}{ Idade (anos) } \\
\hline $18-39$ & $135(91)$ & $14(9)$ & $1,06(0,46 ; 2,45)$ & $0,872^{d}$ & - & \\
\hline$\geq 40$ & $145(90)$ & $16(10)$ & & & & \\
\hline \multicolumn{7}{|c|}{ Raça/cor da pele } \\
\hline Branca & $70(90)$ & $8(10)$ & $0,92(0,37 ; 2,51)$ & $0,859^{d}$ & - & \\
\hline Não branca & $208(90)$ & $22(9 \%)$ & & & & \\
\hline \multicolumn{7}{|c|}{ Chefe da família com tuberculose } \\
\hline Sim & $122(90)$ & $14(10)$ & $1,13(0,492,58)$ & $0,745^{d}$ & - & \\
\hline Não & $158(91)$ & $16(9)$ & & & & \\
\hline \multicolumn{7}{|c|}{ Emprego formal } \\
\hline Sim & $112(93)$ & $9(7)$ & $1,55(0,65 ; 3,99)$ & $0,286^{d}$ & - & \\
\hline Não & $168(89)$ & $21(11)$ & & & & \\
\hline \multicolumn{7}{|c|}{ Abandono escolar ou do trabalho } \\
\hline $\operatorname{Sim}$ & $169(88)$ & $22(12)$ & $1,98(0,78 ; 5,69)$ & $0,121^{d}$ & - & \\
\hline Não & $107(94)$ & $7(6)$ & & & & \\
\hline \multicolumn{7}{|l|}{ Plano de saúde } \\
\hline Sim & $61(92)$ & $5(8)$ & $0,71(0,20 ; 2,02)$ & $0,515^{d}$ & - & \\
\hline Não & $219(90)$ & $25(10)$ & & & & \\
\hline \multicolumn{7}{|c|}{$\begin{array}{l}\text { Nível intermediário: variáveis de informações clínicas do atual tratamento da tuberculose e alterações familiares durante } 0 \text { adoecimento por } \\
\text { tuberculose }\end{array}$} \\
\hline \multicolumn{7}{|c|}{ Tipo de tuberculose } \\
\hline Pulmonar & $208(90)$ & $23(10)$ & $1,13(0,44 ; 3,27)$ & $0,776^{d}$ & - & \\
\hline Extrapulmonar & $72(91)$ & $7(9)$ & & & & \\
\hline \multicolumn{7}{|c|}{ Fase do tratamento } \\
\hline Intensiva & $75(88)$ & $10(12)$ & $1,36(0,54 ; 3,22)$ & $0,445^{d}$ & - & \\
\hline Continuação & $205(91)$ & $20(9)$ & & & & \\
\hline \multicolumn{7}{|l|}{ HIV } \\
\hline Positivo & $50(93)$ & $4(7)$ & $0,69(0,16 ; 2,16)$ & $0,616^{\mathrm{e}}$ & - & \\
\hline Negativo & $190(90)$ & $22(10)$ & & & & \\
\hline \multicolumn{7}{|l|}{ Hospitalização } \\
\hline $\operatorname{Sim}$ & $173(91)$ & $17(9)$ & $1,23(0,52 ; 2,82)$ & $0,584^{d}$ & - & \\
\hline Não & $107(89)$ & $13(11)$ & & & & \\
\hline
\end{tabular}


Continuação

Tabela 2 - Desfecho do tratamento para tuberculose e razão de chances do desfecho desfavorável pelas características dos participantes com tuberculose das cinco capitais ( $n=310)$, Brasil, junho/2016julho/2018

\begin{tabular}{|c|c|c|c|c|c|c|}
\hline \multirow[b]{2}{*}{ Variáveis } & \multicolumn{2}{|c|}{ Desfecho do tratamento } & \multirow{2}{*}{$\begin{array}{c}\text { Razão de chances } \\
\text { bruta } \\
\left(\mathrm{IC}_{95 \%}^{\mathrm{a}}\right)\end{array}$} & \multirow[b]{2}{*}{ p-valor } & \multirow{2}{*}{$\begin{array}{c}\text { Razão de chances } \\
\text { ajustada } \\
\left(\text { IC }_{95 \%}{ }^{a}\right)\end{array}$} & \multirow[b]{2}{*}{ p-valor } \\
\hline & $\begin{array}{c}\text { Favorável } \\
\text { (\%) }\end{array}$ & $\begin{array}{c}\text { Desfavorável } \\
\text { (\%) }\end{array}$ & & & & \\
\hline \multicolumn{7}{|c|}{ Tratamento anterior de tuberculose } \\
\hline $\operatorname{Sim}$ & $43(81)$ & $10(19)$ & $2,75(1,07 ; 6,66)$ & $0,013^{d}$ & $2,34(0,99 ; 5,53)$ & 0,051 \\
\hline Não & $237(92)$ & $20(8)$ & & & 1,00 & \\
\hline \multicolumn{7}{|c|}{ Primeiro atendimento } \\
\hline Público & $254(91)$ & $25(9)$ & $1,19(0,12 ; 5,52)$ & $0,686^{e}$ & - & \\
\hline Privado & $17(89)$ & $2(11)$ & & & & \\
\hline \multicolumn{7}{|c|}{ Comorbidades } \\
\hline Sim & $119(91)$ & $12(9)$ & $0,9(0,38 ; 2,06)$ & $0,792^{\mathrm{d}}$ & - & \\
\hline Não & $161(90)$ & $18(10)$ & & & & \\
\hline \multicolumn{7}{|c|}{ Sequela social } \\
\hline $\operatorname{sim}$ & $182(90)$ & $21(10)$ & $1,25(0,52 ; 3,23)$ & $0,584^{d}$ & - & \\
\hline Não & $98(92)$ & $9(8)$ & & & & \\
\hline \multicolumn{7}{|c|}{ Abandono do estudo } \\
\hline Sim & $5(100)$ & - & $0(0,00 ; 7,2)$ & $0,599^{e}$ & - & \\
\hline Não & $275(90)$ & $30(10)$ & & & & \\
\hline \multicolumn{7}{|c|}{ Abandono do trabalho } \\
\hline Sim & $80(86)$ & $13(14)$ & $1,91(0,81 ; 4,38)$ & $0,094^{d}$ & - & \\
\hline Não & $200(92)$ & $17(8)$ & & & & \\
\hline \multicolumn{7}{|l|}{ Divórcio } \\
\hline Sim & $8(67)$ & $4(33)$ & $5,23(1,06 ; 21,00)$ & $0,02^{\mathrm{e}}$ & $5,29(1,39 ; 20,05)$ & 0,014 \\
\hline Não & $272(91)$ & $26(9)$ & & & 1,00 & \\
\hline \multicolumn{7}{|c|}{ Perturbação da vida sexual } \\
\hline Sim & $10(100)$ & - & $0(0,00 ; 3,54)$ & $0,606^{e}$ & - & \\
\hline Não & $270(90)$ & $30(10)$ & & & & \\
\hline \multicolumn{7}{|c|}{ Adoecimento de filho } \\
\hline Sim & $4(100)$ & - & $0(0,00 ; 9,14)$ & $0,664^{e}$ & - & \\
\hline Não & $276(90)$ & $30(10)$ & & & & \\
\hline \multicolumn{7}{|c|}{ Afastamento das pessoas } \\
\hline $\operatorname{Sim}$ & $72(92)$ & $6(8)$ & $0,72(0,23 ; 1,91)$ & $0,493^{d}$ & - & \\
\hline Não & $208(90)$ & $24(10)$ & & & & \\
\hline \multicolumn{7}{|l|}{ Depressão } \\
\hline Sim & $9(100)$ & - & $0(0,00 ; 3,96)$ & $0,395^{\mathrm{e}}$ & - & \\
\hline Não & $271(90)$ & $30(10)$ & & & & \\
\hline \multicolumn{7}{|c|}{ Experiência de preconceito devido à tuberculose } \\
\hline $\operatorname{Sim}$ & $15(94)$ & $1(6)$ & $0,60(0,01 ; 4,25)$ & $0,528^{e}$ & - & \\
\hline Não & $265(90)$ & $29(10)$ & & & & \\
\hline
\end{tabular}

a) $I C_{95 \%}$ : intervalo de confiança de $95 \%$; b) Teste de Wald; c) HIV:vírus da imunodeficiência humana (sigla em inglês para human immunodeficiency virus); d) Teste qui-quadrado de Pearson;e) Teste exato de Fisher. 
Continuação

\section{Tabela 2 -Desfecho do tratamento para tuberculose e razão de chances do desfecho desfavorável pelas características dos participantes com tuberculose das cinco capitais $(n=310)$, Brasil, junho/2016- julho/2018}

\begin{tabular}{|c|c|c|c|c|c|c|}
\hline \multirow[b]{2}{*}{ Variáveis } & \multicolumn{2}{|c|}{ Desfecho do tratamento } & \multirow{2}{*}{$\begin{array}{c}\text { Razão de chances } \\
\text { bruta } \\
\left(\mathrm{IC}_{95 \%}{ }^{\mathrm{a}}\right)\end{array}$} & \multirow[b]{2}{*}{ p-valor } & \multirow{2}{*}{$\begin{array}{c}\text { Razão de chances } \\
\text { ajustada } \\
\left(\mathrm{IC}_{95 \%}{ }^{\mathrm{a}}\right)\end{array}$} & \multirow[b]{2}{*}{ p-valor ${ }^{b}$} \\
\hline & $\begin{array}{c}\text { Favorável } \\
\text { (\%) }\end{array}$ & $\begin{array}{c}\text { Desfavorável } \\
\text { (\%) }\end{array}$ & & & & \\
\hline \multicolumn{7}{|c|}{ Outras sequelas sociais } \\
\hline $\operatorname{Sim}$ & $102(92)$ & $8(7)$ & $0,63(0,23 ; 1,54)$ & $0,288^{d}$ & - & \\
\hline Não & $178(89)$ & $22(11)$ & & & & \\
\hline \multicolumn{7}{|c|}{ Nível proximal: variáveis financeiras } \\
\hline \multicolumn{7}{|c|}{ Custo catastrófico } \\
\hline $\operatorname{sim}$ & $109(85)$ & $19(15)$ & $2,70(1,16 ; 6,54)$ & $0,010^{\mathrm{d}}$ & $2,53(1,13 ; 5,67)$ & 0,023 \\
\hline Não & $171(94)$ & $11(6)$ & & & 1,00 & \\
\hline \multicolumn{7}{|c|}{ Condição financeira do domicílio antes da tuberculose } \\
\hline Pobre & $74(85)$ & $13(15)$ & $2,12(0,90 ; 4,89)$ & $0,050^{d}$ & - & \\
\hline Não pobre & $206(92)$ & $17(8)$ & & & & \\
\hline \multicolumn{7}{|c|}{ Condição financeira do domicílio depois da tuberculose } \\
\hline Pobre & $104(87)$ & $16(13)$ & $1,93(0,84 ; 4,46)$ & $0,084^{d}$ & - & \\
\hline Não pobre & $176(93)$ & $14(7)$ & & & & \\
\hline \multicolumn{7}{|c|}{ Alteração de renda do domicílio após a tuberculose } \\
\hline $\operatorname{Sim}$ & $33(92)$ & $3(8)$ & $0,83(0,15 ; 2,94)$ & $0,529^{e}$ & - & \\
\hline Não & $247(90)$ & $27(10)$ & & & & \\
\hline Total & $280(90)$ & $30(10)$ & & & & \\
\hline
\end{tabular}

a) $\mathrm{IC}_{955}$ : intervalo de confiança de $95 \%$; b) Teste de Wald; c) HIV:vírus da imunodeficiência humana (sigla em inglês para human immunodeficiency virus); d) Teste qui-quadrado de Pearson; e) Teste exato de Fisher.

período do adoecimento, sendo importante considerar a necessidade de intervenção governamental com o propósito de minimizar os custos catastróficos relacionados à tuberculose, mediante política pública específica, de forma a reduzir ou mesmo evitar a perda de renda e, assim, melhorar os indicadores de desfecho do tratamento. Esse mecanismo de enfrentamento, comumente sugerido, pode aliviar o fardo financeiro relatado em outros estudos e otimizar a cura da tuberculose nessa população. ${ }^{1-4,16}$

A perda do emprego e da renda, após o diagnóstico da doença, são extremamente importantes como fatores determinantes para o empobrecimento do domicílio, haja vista os indivíduos não conseguirem manter a estabilidade econômica da família após adoecerem. 0 estudo observou que $11 \%$ dos domicílios sofreram importante alteração de renda, se compararmos os períodos anterior e posterior ao adoecimento, levando ao empobrecimento. Os achados deste trabalho corroboram os de estudos realizados na Indonésia em 2018 e 2019, onde se constatou a relevância da prestação de auxílio para viagens e apoio nutricional, e, ademais, proteção social ao enfermo, familiares e pessoas mais próximas a ele, afetados pelos custos da tuberculose no contexto de seu domicílio. ${ }^{4,18}$

Achados importantes descrevem a desestruturação ou ruptura do domicílio de um doente por tuberculose, fato todavia negligenciado nos estudos conhecidos. Divórcio ou separação do parceiro e perturbação da vida sexual, sequelas sociais presentes entre os participantes desta pesquisa, são exemplos disso. 0 trabalho em tela mostrou que experimentar divórcio devido ao adoecimento aumentou em cerca de cinco vezes as chances de desfecho desfavorável do tratamento. Em 2016, foram registrados 344.526 divórcios no Brasil - 2,38 divórcios por 1 mil habitantes, frequência inferior à observada aqui. ${ }^{28}$

Os determinantes do desfecho do tratamento revelados neste estudo destacam a magnitude do problema da perda de renda e a necessidade de abordá-la de maneira adequada, além das dificuldades sociais enfrentadas pela condição de tuberculose. 
Os domicílios, tanto pobres como não pobres, correm o risco de experimentar a pobreza mais profundamente. Este risco é mais evidente em participantes de famílias pobres. ${ }^{18}$ Os achados do estudo sugerem que outras medidas, além dos serviços médicos gratuitos, sejam consideradas, principalmente quando se destinam a pessoas no limiar da pobreza.

Mais da metade dos indivíduos com tuberculose entrevistados tem vivenciado alguma sequela social, aproximadamente um quarto experimentou abandono do trabalho e um quarto mencionou afastamento por outras pessoas. Em proporções distintas às identificadas em outras pesquisas, muito embora justamente pela semelhança experimentada, cumpre considerar diferenças socioeconômicas e de sistemas de saúde entre diferentes países, suas respectivas especificidades e fatores determinantes da evolução do indivíduo com tuberculose e seu impacto no próprio domicílio. ${ }^{1,3}$

\section{Referências}

1. Nhung NV, Hoa NB, Anh NT, Ngoc Anh LT, Siroka A, Lönnroth K, et. al. Measuring catastrophic costs due to tuberculosis in Viet Nam. Int J Tuberc Lung Dis. 2018 Sep 1;22(9):983-90. doi: http://doi. org/10.5588/ijtld.17.0859.

2. Pedrazzoli D, Siroka A, Boccia D, Bonsu F, Nartey K, Houben R, et al. How affordable is TB care? Findings from a nationwide TB patient cost survey in Ghana. Trop Med Int Health. 2018;23(8):870-8. doi: https:// doi.org/10.1111/tmi.13085.

3. Muttamba W, Tumwebaze R, Mugenyi L, Batte C, Sekibira R, Nkolo A, et al. Households experiencing catastrophic costs due to tuberculosis in Uganda: magnitude and cost drivers. BMC Public Health. 2020 Sep 16;20(1):1409. doi: https://doi.org/10.1186/ s12889-020-09524-5.

4. Fuady A, Houweling TAJ, Mansyur M, Burhan E, Richardus JH. Effect of financial support on reducing the incidence of catastrophic costs among tuberculosis-affected households in Indonesia: eight simulated scenarios. Infect Dis Poverty. $2019 \mathrm{Feb}$ 2;8(1):10. doi: https://doi.org/10.1186/s40249-0190519-7.

5. Hargreaves JR, Boccia D, Evans, CA, Adato M, Petticrew M, Porter JDH. Os determinantes sociais da tuberculose: das evidências à ação. Am J Public Health. 2011;101(4):654-62. doi: https://doi. org/10.2105/AJPH.2010.199505.
Em conclusão, as famílias brasileiras correm 0 risco de enfrentar dificuldades financeiras durante 0 adoecimento de um de seus membros por tuberculose, capazes de afetar o desfecho de seu tratamento.

\section{Contribuições dos autores}

Guidoni LM, Maciel ELN e Zandonade E contribuíram com atividades substanciais à concepção do projeto, aquisição, análise e interpretação de dados, redação e revisão crítica de conteúdo intelectual relevante do trabalho. Fregona GC, Negri LSA, Oliveira SMVL, Prado TN, Salles CMM, Coimbra RS e Galavote HS contribuíram para a interpretação de dados e revisão crítica do conteúdo intelectual do trabalho. Todos os autores aprovaram a versão final do manuscrito e são responsáveis por todos os seus aspectos, incluindo a garantia de sua precisão e integridade.

6. Oxlade 0, Murray M. Tuberculosis and poverty: why are the poor at greater risk in India? PLoS One. 2012;7(11):e47533. doi: https://doi.org/10.1371/ journal.pone.0047533.

7. World Health Organization. WHO Report 2016: global tuberculosis control. Geneva: WHO: 2017.

8. World Health Organization. WHO Report 2015: global tuberculosis control: surveillance, planning, financing. Geneva: WHO: 2015.

9. World Health Organization. WHO report 2019: global tuberculosis control: surveillance, planning, financing. Geneva: WHO; 2019.

10. Ministério da Saúde (BR), Secretaria de Vigilância em Saúde. Doença pelo coronavírus: COVID-19. [Brasília, DF]: MS; 2020. (Boletim epidemiológico especial: $n$. 33).

11. Haling G, Castro MC. A spatial analysis of social and economic determinants of tuberculosis in Brazil. Health Place. 2014;25:56-67. doi: https://doi. org/10.1016/j.healthplace.2013.10.008.

12. World Health Organization. The end tuberculosis strategy. Geneva: WHO; 2016.

13. World Health Organization. Tuberculosis patient cost surveys: a handbook. Geneva: WHO; 2017.

14. World Health Organization. Protocol for survey to determine direct and indirect costs due to 
tuberculosis and to estimate proportion of tuberculosis-affected households experiencing catastrophic total costs due to tuberculosis. Geneva: WHO; 2015.

15. Ministério da Saúde (BR), Secretaria de Vigilância em Saúde. Manual de recomendações para o controle da tuberculose no Brasil. Brasília, DF: MS; 2019.

16. Tanimura T, Jaramillo E, Well D, Raviglione M, Lonnroth K. Financial burden for tuberculosis patients in low- and middle-income countries: a systematic review. Eur Respir J. 2015;43(6):1763-75. doi: https://doi.org/10.1183/09031936.00193413.

17. Jackson S, Sleigh AC, Wang GJ, Liu XL. Poverty and the economic effects of tuberculosis in rural China. Int J Tuberc Lung Dis. 2006:10(10):1104-10.

18. Fuady A, Houweling TAJ, Mansyur M, Richardus JH. Catastrophic total costs in tuberculosis-affected households and their determinants since Indonesia's implementation of universal health coverage. Infect Dis Poverty. 2018 Jan. 12;7(1):3. doi: https://doi. org/10.1186/s40249-017-0382-3.

19. Steffen R, Menzies D, Oxlade O, Pinto M, Castro AZ, Monteiro P, et al. Patients' costs and cost-effectiveness of tuberculosis treatment in DOTS and non-DOTS facilities in Rio de Janeiro, Brazil. PLoS One. 2010 Nov 17;5(11):e14014. doi: https://doi.org/10.1186/ s40249-017-0382-3.2018.

20. Costa JG, Santos AC, Rodrigues LC, Barreto ML, Roberts JA. Tuberculosis in Salvador, Brazil: costs to health system and families. Rev Saude Publica. 2005;39(1):122-8. doi: http://doi.org/10.1590/ s0034-89102005000100016. Português

21. Prado TN, Wada N, Guidoni LM, Golub JE, Dietze R, Maciel ELN. Cost-effectiveness of community health worker versus home-based guardians for directly observed treatment of tuberculosis in Vitoria,
Espírito Santo State, Brazil. Cad Saude Publica. 2011;27(5):944-52. doi: http://doi.org/10.1590/ s0102-311x2011000500012.

22. KNCV Tuberculosis Foundation. Tool to estimate patients' costs literature review. [The Hague, NL: KNCV]; 2008.

23. Nunes GF, Guidoni LM, Negri LSA, Zandonade E, Maciel ELN. Adaptação transcultural do instrumento "Tool to estimate patient's costs" em municípios prioritários do Brasil no controle da tuberculose. Esc Anna Nery. 2018;22(4):e20180073. doi: http://doi. org/10.1590/2177-9465-ean-2018-0073.

24. Silva EM, Silva MT, Pereira MG. Identificação, mensuração e valoração de custos em saúde. Epidemiol Serv Saude. 2016;25(2):43739. doi: http://dx.doi.org/10.5123/S167949742016000200023 .

25. Instituto Brasileiro de Geografia e Estatística. Síntese de indicadores sociais: uma análise das condições de vida da população brasileira: Coordenação de População e Indicadores Sociais. Rio de Janeiro: IBGE; 2017.

26. Wingfield T, Tovar MA, Huff D, Boccia D, Montoya R, Ramos E, et. al.. The economic effects of supporting tuberculosis-affected households in Peru. Eur Respir J. 2016;48(5):1396-410. doi: http://dx.doi. org/10.1183/13993003.00066-2016.

27. Tomeny EM, Mendoza VL, Marcelo DB, Barrameda AJD, Langley I, Abong JM, et al. Patient-cost survey for tuberculosis in the context of patient-pathway modelling. Int J Tuberc Lung Dis. 2020:24(4):420-7. doi: http://doi.org/10.5588/ijtld.19.0206.

28. Instituto Brasileiro de Geografia e Estatística. Informativo das estatísticas do registro civil. Rio de Janeiro: IBGE; 2016. 8 P. (vol. 43). 


\section{Abstract}

Objective: To evaluate the impact of catastrophic costs on unfavorable tuberculosis treatment outcomes. Methods: This was a prospective cohort study, conducted in five Brazilian state capitals (Manaus, Recife, Vitória, Campo Grande and Porto Alegre) from June 2016 to July 2018. Logistic regression was used to calculate the odds ratio (OR) and $95 \%$ confidence intervals (95\% CI). Results: Of the 350 participants, 310 were included, of whom 30 presented unfavorable outcomes. Catastrophic cost (OR=2.53 -95\% CI 1.13;5.67) and divorce (OR=5.29 -95\% CI 1.39;20.05) increased the chances of unfavorable outcomes. Conclusion: Financial difficulties during tuberculosis treatment may impair its outcome. Catastrophic cost and divorce were determining factors for treatment outcomes.

Keywords: Tuberculosis; Therapeutics; Costs and Cost Analysis; Cohort Studies; Socioeconomic Factors; Brazil.

\section{Resumen}

Objetivo: Evaluar el impacto de los costos catastróficos en el resultado desfavorable del tratamiento de la tuberculosis. Métodos: Estudio de cohorte prospectiva realizado en cinco capitales de estados brasileños (Manaus, Recife, Vitória, Campo Grande y Porto Alegre) entre junio de 2016 y julio de 2018. Se utilizó la regresión logística para calcular los odds ratios (OR) y los intervalos de confianza del 95\% (IC95\%). Resultados: De los 350 participantes, se incluyeron 310, y 30 tuvieron resultado desfavorable. El costo catastrófico $(O R=2,53$ - IC95\% 1,13;5,67) y el divorcio $(O R=5,29-I C 95 \%$ 1,39;20,05) aumentaron la obtención de un resultado desfavorable. Conclusión: Dificultades económicas enfrentadas durante el tratamiento de la tuberculosis pueden perjudicar el resultado del tratamiento. Las determinantes del tratamiento fueron el costo catastrófico y el divorcio.

Palabras clave: Tuberculosis; Terapéutica; Costos y Análisis de Costo; Estudios de Cohortes; Factores Socioeconómicos; Brasil.

Recebido em 27/09/2020

Aprovado em 09/02/2021

Editora associada:Taís Freire Galvão - (D orcid.org/0000-0003-2072-4834 Editora científica:Taís Freire Galvão - (D orcid.org/0000-0003-2072-4834 Editora geral: Leila Posenato Garcia - (D orcid.org/0000-0003-1146-2641 


\section{Errata}

No artigo "Custos catastróficos e sequelas sociais decorrentes do diagnóstico e tratamento da tuberculose no Brasil", com número de doi: 10.1590/\$1679-49742021000300012, publicado no periódico Epidemiologia e Serviços de Saúde, 30(3):1-14, na página 1:

Onde se lia:

Márcia Regina de Oliveira Pedroso ${ }^{1}$ - ๑ orcid.org/0000-0002-2859-159X

Leticia Molino Guidoni ${ }^{1}$ - ๑ orcid.org/0000-0003-3737-5407

Eliana Zandonade ${ }^{2}$ - ๑ orcid.org/0000-0001-5160-3280

Geisa Fregona ${ }^{2}$ - ๑ orcid.org/0000-0001-5835-8222

Letícya dos Santos Almeida Negri ${ }^{1}$ - ๑ orcid.org/0000-0002-0056-4506

Sandra Maria do Valle Leone de Oliveira ${ }^{3}$ - ๑ orcid.org/0000-0002-8960-6716

Thiago Nascimento do Prado ${ }^{1}$ - ๑ orcid.org/0000-0001-8132-6288

Carolina Maia Martins Sales ${ }^{1}$ - ๑ orcid.org/0000-0002-2879-5621

Raisa da Silveira Coimbra ${ }^{1}$ - ๑ orcid.org/0000-0003-2528-6044

Heletícia Scabelo Galavote ${ }^{4}$ - ๑ orcid.org/0000-0003-4490-6763

Ethel Leonor Noia Maciel $^{2}$ - ๑ orcid.org/0000-0003-4826-3355

Leia-se:

Leticia Molino Guidoni ${ }^{1}$ - ๑ orcid.org/0000-0003-3737-5407

Eliana Zandonade ${ }^{2}$ - ๑ orcid.org/0000-0001-5160-3280

Geisa Fregona $^{2}$ - ๑ orcid.org/0000-0001-5835-8222

Letícya dos Santos Almeida Negri ${ }^{1}$ - ๑ orcid.org/0000-0002-0056-4506

Sandra Maria do Valle Leone de Oliveira ${ }^{3}$ - ๑ orcid.org/0000-0002-8960-6716

Thiago Nascimento do Prado ${ }^{1}$ - ๑ orcid.org/0000-0001-8132-6288

Carolina Maia Martins Sales ${ }^{1}$ - ๑ orcid.org/0000-0002-2879-5621

Raisa da Silveira Coimbra ${ }^{1}$ - ๑ orcid.org/0000-0003-2528-6044

Heletícia Scabelo Galavote ${ }^{4}$ - ๑ orcid.org/0000-0003-4490-6763

Ethel Leonor Noia Maciel $^{2}$ - ๑ orcid.org/0000-0003-4826-3355 